\title{
The Complete Set of Hamiltonian Intermittency Scaling Behaviors
}

\author{
Albert B. Zisook \\ Lyman Laboratory of Physics, Harvard University, Cambridge, MA 02138, USA
}

\begin{abstract}
Renormalization group equations describing the phenomenon of intermittency in Hamiltonian systems are presented. All solutions satisfying certain physical constraints are obtained; they are the complete set of simple singularities. Further considerations lead to precise predictions for scaling behavior at the onset of intermittency.
\end{abstract}

\section{Introduction}

It has long been known that a Hamiltonian system with one degree of freedom, $H(p, q)$, has particularly simple behavior near an elliptic or hyperbolic fixed point. A canonical transformation achieves Birkhoff's normal form

$$
H(p, q)=a\left(\frac{p^{2}+q^{2}}{2}\right)+b\left(\frac{p^{2}+q^{2}}{2}\right)^{2}+c\left(\frac{p^{2}+q^{2}}{2}\right)^{2}+\cdots
$$

in the neighborhood of an elliptic fixed point, or the normal form

$$
H(p, q)=a p q+b(p q)^{2}+c(p q)^{3}+\cdots
$$

in the neighborhood of a hyperbolic fixed point $[1,2]$. What is the corresponding normal form in the limit of marginal stability $(a=0)$ ? An answer to this question is found in the singularity theory of Arnold [3]. By limiting consideration to a special type of behavior, called "simple," he obtains a discrete classification of the possibilities. The results are not widely known or understood by physicists, perhaps because of the unfamiliar mathematical techniques involved. Presented in this paper is a physically motivated calculation of Arnold's simple normal forms, based on the renormalization group for mappings introduced by Feigenbaum [4,5].

Feigenbaum's renormalization group for mappings has been used to study three phenomena occurring in Hamiltonian systems: infinite cascades of period-doubling bifurcations, the breakup of KAM tori and tangent bifurcations [6-11, 23-25]. The classification of simple normal forms is obtained through a comprehensive treatment of the latter. The physical motivation for studying tangent bifurcations is 
that they provide a setting for a theoretical description of intermittency, a behavior generally characterized by chaotic or irregular interruptions to an otherwise orderly or regular motion. Pomeau and Manneville have suggested that this behavior may be intimately tied to the local disappearance of periodic motion which may occur in such a bifurcation $[12,13]$. They suggest that trajectories may spend a long time in the region of phase space from which a periodic orbit has disappeared before finally wandering away. If there is no attracting region elsewhere, it is reasonable to assume that the trajectory will eventually return to this special region and orbit in a regular fashion for another relatively long time. This repeated alternation between nearly periodic orbiting and disorderly wandering describes qualitatively and quantitatively the intermittency observed in some electrical circuits and hydrodynamical systems $[14,15]$.

Later, it was observed that the calculations of Pomeau and Manneville could be performed within the renormalization group formalism of Feigenbaum [16-18]. Further research showed that these results for dissipative systems (as represented by one-dimensional maps) had analogs for Hamiltonian systems (as represented by area-preserving two-dimensional maps) [9-11]. However, the problem has yet to be treated in generality sufficient to obtain all solutions. In Sect. II, a derivation of the appropriate generalization of Feigenbaum's equation will be presented. In Sect. III, the set of all solutions satisfying several physically motivated constraints are calculated (they turn out to be Arnold's simple singularities). The last section concerns the physical predictions which follow from the analysis.

It is important to emphasize the differences between the present treatment and the work of Arnold. While Arnold classified critical points of real-valued functions of $n$ real variables, the treatment here is formulated in terms of Hamiltonians with one degree of freedom, which may be thought of as functions of two variables. For this reason, the result in this paper is less general. However, it is precisely this limitation which makes a direct calculation possible. It is hoped that Arnold's list of normal forms will appear less obscure when it arises more naturally in this different setting.

\section{The Renormalization Equations}

An area-preserving mapping or canonical transformation of one degree of freedom may be relevant in several physical situations. Such a mapping may arise as the Poincaré section of a Hamiltonian with two degrees of freedom, a description of lines of magnetic flux in a Tokamak or a model for the beam-beam interaction in a particle accelerator [19-21]. Integration of a Hamiltonian flow, such as that induced by $H(p, q)$, for a fixed amount of time also yields a canonical transformation.

In order for a periodic orbit to destabilize and disappear, certain local conditions must be satisfied. It is sufficient to study the disappearance of fixed points, rather than periodic orbits, as each element of a cycle of length $I$ is a fixed point of the mapping iterated I times. The inverse function theorem guarantees that a fixed point depends continuously on parameters in the system whenever the linear stability matrix of the mapping has no eigenvalues equal to one. For area-preserving mappings of the plane, the product of the eigenvalues obtained in a linear stability analysis is always equal to one. Thus a necessary condition for the disappearance of 
a fixed point of a canonical transformation is that the linear stability matrix have both eigenvalues equal to one.

The property of a mapping, $T$, relevant to intermittency can be condensed into a single quantity whose calculation is the object of theory. This quantity, called $\tau(p, q)$, is defined as the number of iterations for which an orbit passing through $(p, q)$ is contained inside some small fixed ball around the fixed point, henceforth taken to be the origin. In the limit $p, q \rightarrow 0, \tau$ will diverge and may be considered a continuous function of its arguments. The possible asymptotic behaviors of $\tau(p, q)$ in this limit may be obtained using a renormalization group.

By removing irrelevant information, the repeated renormalization of $T$ yields a canonical transformation with special properties. The first step in the renormalization procedure is to change the scale

$$
T \rightarrow T^{\prime} \equiv L T L^{-1}
$$

Here $L$ is a $2 \times 2$ matrix of constants whose eigenvalues lie outside the unit circle. The effect of repeating this operation is to reduce any mapping to a linear one. In the case of an elliptic on hyperbolic fixed point the linear part provides a good description of the motion, being a rotation or dilatation, respectively. In the marginal case, however, one renormalizes to the matrix $\left(\begin{array}{ll}1 & A \\ 0 & 1\end{array}\right)$ (possibly with $A=0$ ). Too much information is lost in this process, so a compensating step in the renormalization is necessary. To balance the slowing down of orbits scaled towards the origin, the map is iterated $k$ times

$$
T \rightarrow\left(T^{\prime}\right)^{k}=\left(L T L^{-1}\right)^{k}=L T^{k} L^{-1} .
$$

The effect of this iteration on the basic quantity $\tau(p, q)$ is merely to divide it by $k$, leaving details of the dependence on $p$ and $q$ unchanged. A priori, it is not clear that this simple renormalization (merely a change of scale in phase space and time) will have interesting fixed points. It turns out to be so. Note that for $k=2$ this is precisely Feigenbaum's functional equation [9-11, 16-18].

If a canonical transformation $T$ is fixed by the renormalization it must also satisfy the more general relation

$$
T=L^{n} T^{k^{n}} L^{-n}
$$

This means that $T$ has a $k^{n}$-th root, namely $L^{n} T L^{-n}$. As $k^{n} \rightarrow \infty$ this approaches the identity, encouraging the assumption that it may be written as the exponential of some vector field. [Area-preserving mappings form a Lie group; divergence-free vector fields in the plane form the corresponding Lie algebra. In finite-dimensional Lie groups the exponential map is onto some neighborhood of the identity. Assuming this result in the infinite-dimensional case at hand results in exponentially small errors which will not affect the results of this paper which apply in the scaling $\operatorname{limit}(p, q) \rightarrow 0$.] If this $k^{n}$-th root is an exponential, $n$ may be considered a continuous variable, rather than integral. Here lies the difference between intermittency fixed points and Feigenbaum's fixed points describing period-doubling cascades. The period-doubling fixed point is a mapping which does not fix the origin, and consequently, its $2^{n}$-th roots map the origin yet further as $n$ increases. Thus these 
transformations do not become infinitesimal in the limit $n \rightarrow \infty$, and $n$ cannot be made continuous.

The continuity of $n$ permits the fixed point equation to be reexpressed in terms of vector fields rather than diffeomorphisms. This process corresponds roughly to "taking logs." It is easiest to begin with the correct relation between vector fields and exponentiate. The interest here is in obtaining the correct infinitesimal form of Feigenbaum's equation. The precise conditions for the equivalence of the discrete and continuous formulation will not be discussed. So, let $l$ and $v$ be vector fields in the plane such that

$$
[l, v]=v,
$$

where the bracket denotes the commutator of vector fields. Differentiation yields directly that

$$
\frac{d}{d s}\left(e^{s l} v e^{-s l}\right)=\left(e^{s l}(l v-v l) e^{-s l}\right)=e^{s l} v e^{-s l}
$$

Upon integrating with respect to $s$ one obtains

$$
e^{s l} v e^{-s l}=e^{s} v .
$$

By exponentiation and conjugation (the two commute) an analog of Eq. (5) is obtained

$$
e^{v}=e^{-s l} e^{e^{s} v} e^{s l} .
$$

The analogy is complete with the identifications $T=e^{v}$ and $L=e^{l}$ (the apparent discrepancy in signs is due to the fact that operators compose differently than mappings). The property that $T$ is canonical or area-preserving translates into the requirement that $v$ be divergence-free, therefore of the form

$$
v=\frac{\partial H}{\partial p} \frac{\partial}{\partial q}-\frac{\partial H}{\partial q} \frac{\partial}{\partial p}
$$

for some function $H(p, q)$. By choosing $H(0,0)=0$ the origin becomes a degenerate critical point of the function $H(p, q)$. For $L$ to be a linear transformation with eigenvalues outside the unit circle, the vector field $l$ must be of the form

$$
l=(a p+b q) \frac{\partial}{\partial p}+(c p+d q) \frac{\partial}{\partial q}
$$

with eigenvalues in the right half-plane.

After the introduction of the function $H(p, q)$, whose Hamiltonian flow generates $T$, another simplifying transformation of the fixed point equation is possible. This transformation is accomplished by means of the identities

$$
\begin{aligned}
& ([l, v]-v) p=-l \frac{\partial H}{\partial q}-b \frac{\partial H}{\partial p}+a \frac{\partial H}{\partial q}+\frac{\partial H}{\partial q}=\frac{\partial}{\partial q}(-l H+(1+a+d) H), \\
& ([l, v]-v) q=l \frac{\partial H}{\partial p}-d \frac{\partial H}{\partial p}+c \frac{\partial H}{\partial q}-\frac{\partial H}{\partial p}=\frac{\partial}{\partial p}(l H-(1+a+d) H) .
\end{aligned}
$$


These relations imply that $[l, v]=v$ if and only if $l H=(1+a+d) H$. The fixed point equation is now written in its final form

$$
l H=(1+\operatorname{trace} l) H \equiv \lambda H .
$$

The meaning of Eq. (9) is that orbits scaled by $e^{s l}$ evolve $e^{s}$ times more slowly; in terms of $\tau(p, q)$ this means that

$$
e^{s l} \tau(p, q)=e^{-s} \tau(p, q) .
$$

Or, upon differentiation with respect to $s$,

$$
l \tau=-\tau .
$$

Combining Eq. (13) and (15) it may be concluded that

$$
l\left(H \tau^{\lambda}\right)=0 .
$$

Since $\tau(p, q)$ may depend only on orbits, and not on $p$ and $q$ individually, it must locally be a function of $H(p, q)$. Thus the formula for $\tau$

$$
\tau(p, q) \propto H(p, q)^{-1 / \lambda} .
$$

Thus the functional renormalization equation has been reduced to a simple linear form, which involves precisely those variables necessary for the calculation of the fundamental quantity $\tau(p, q)$.

\section{The Renormalization Fixed Points}

Why should one expect the leading terms of a degenerate Hamiltonian to be the scale-invariant solutions of Eq. (13)? It is easier to understand when one should not expect this scale invariance. Imagine that as parameters in $H(p, q)$ were varied, both the quantities $a$ and $b$ (of Eq. (1) or (2)) tended towards zero simultaneously. Then the limiting value of the ratio $a / b$ would determine properties of the leading behavior "at criticality." This quantity would serve to set a scale of length in phase space, breaking the scale invariance. Thus only those cases where $b$ remains non-zero as $a$ vanishes may be expected to exhibit scaling. Intuitively, the leading behaviors of interest are "smaller" than the term with coefficient $a$ while being "larger" than the term with coefficient $b$. Note that the canonical (but complex) transformation

$$
\left(\begin{array}{l}
p \\
q
\end{array}\right) \rightarrow \frac{1}{\sqrt{2}}\left(\begin{array}{rr}
1 & -i \\
i & 1
\end{array}\right)\left(\begin{array}{l}
p \\
q
\end{array}\right)
$$

transforms Eq. (1) into Eq. (2) and vice versa. This formal equivalence insures that the notion of being "between" the leading two terms does not depend on whether $a=0$ is approached from the elliptic or hyperbolic side. This condition on the size of scaling solutions will lead to the requirement that $\lambda$ be greater than two.

Before solving Eq. (13), it is useful to study briefly the effects of perturbations. The question is: how do small perturbations $\Delta T$ to a scaling canonical transformation $T$ grow upon renormalization?

$$
T+\Delta T \rightarrow L(T+\Delta T)^{k} L^{-1} \equiv T+\Delta T^{\prime} .
$$


It is difficult to perform this calculation in terms of canonical transformations, but very simple when formulated in terms of the Hamiltonians whose flows generate them. In these terms, the renormalization is simply

$$
H(p, q) \rightarrow e^{s \lambda} e^{-s l} H(p, q) \equiv H^{\prime}(p, q) .
$$

The operator $e^{-s l}$ changes the scale in phase space and the operator $e^{s \lambda}$ changes the scale of time. By solving

$$
(\lambda-l) \Delta H=\Lambda \Delta H,
$$

the eigenperturbations $\Delta H$ and their eigenvalues $\Lambda$ may be found. Upon renormalization, the perturbation $\Delta H$ grows as $e^{s \Lambda}$, and hence is said to be relevant, marginal or irrelevant when $\Lambda$ is respectively positive, zero or negative.

Now the scaling equation (13) may be attacked directly. The first step in solution is the diagonalization of the operator $l$ by a linear canonical change of coordinates, so that

$$
l=\alpha p \frac{\partial}{\partial p}+\delta q \frac{\partial}{\partial q} .
$$

Then, if

$$
H(p, q)=\sum_{n, m} A_{n m} p^{n} q^{m},
$$

the scaling equation (13) says that $A_{n m}=0$, unless

$$
n \alpha+m \delta=1+\alpha+\delta \equiv \lambda .
$$

In other words, the allowed values of $m$ and $n$ lie along a line in the space of pairs $(m, n)$.

In these coordinates, every monomial $p^{n} q^{m}$ is an eigenperturbation with eigenvalue $\Lambda=\lambda-n \alpha-m \delta$. In particular, the perturbation $p^{2} q^{2}$ has eigenvalue $\Lambda=\lambda-2 \alpha-2 \delta=2-\lambda$ and is irrelevant only when $\lambda>2$.

In canonically equivalent coordinates the perturbation $p^{2} q^{2}$ becomes $\left(p^{2}+q^{2}\right)^{2}$, which is therefore irrelevant under the same condition: that $\lambda>2$. So whenever $\lambda>2$, the scaling terms (which are marginal) will be "bigger" than the term with coefficient $b$ in the Birkhoff expansion (which is irrelevant). This restrictions forces $\alpha$ and $\delta$ to be real. For if $\alpha=\bar{\delta}$ is complex, then $n$ must be equal to $m$ in Eq. (23). From this would follow the contradiction $\lambda=n /(n-1) \leqq 2$. Since $\alpha$ and $\delta$ are real and in the right half-plane they are positive.

There is one final technical restriction on solutions to the scaling equation. The flow of $H(p, q)$ must not have curves consisting entirely of fixed points. Such a Hamiltonian describes a tangent bifurcation in which a non-isolated fixed point appears. Although theoretically possible, these solutions will be rejected as unphysical.

The solutions are obtained by drawing lines $\alpha n+\delta m=\lambda$ in $(m, n)$-space. Such lines divide the quadrant $m, n \geqq 0$ into distinct regions. Lattice points $(m, n)$ in the bounded region correspond to irrelevant perturbations $(\Lambda=\lambda-\alpha n-\delta m>0)$. Those in the unbounded region correspond to irrelevant perturbations $(\Lambda=$ $\lambda-\alpha n-\delta m<0$ ). Lattice points on the line $\alpha n+\delta m=\lambda$ are clearly marginal. 


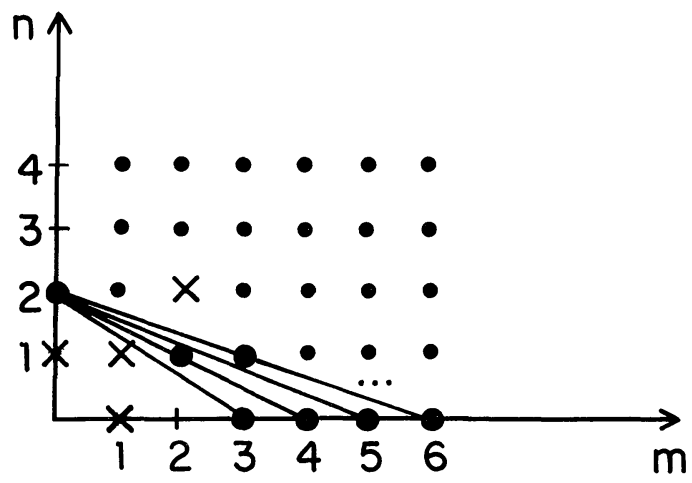

Fig. 1. Arnold's simple singularities $A_{N}$

The restrictions discussed above have simple geometrical interpretations. If $p^{2} q^{2}$ is to be irrelevant, the point $m=n=2$ must lie in the unbounded region. If the line $p=0$ is not to consist entirely of fixed points, $H(p, q)$ must contain some term $p^{n} q^{m}$ with $n<2$. Similarly, $H(p, q)$ must contain some term with $m<2$.

It is now easy to classify the solutions. Acceptable lines in the $(m, n)$-plane must contain a point with $m<2$ as well as one with $n<2$. The points $(1,0),(0,1)$ and $(1,1)$ must always lie in the bounded region because $\Lambda=1+\alpha+\beta-n \alpha-m \beta>0$ for any $\alpha, \beta>0$. Thus if $(2,2)$ is to be in the unbounded region, the line must pass through at least one of the points $(2,0),(0,2),(2,1),(1,2),(3,0)$ or $(0,3)$. Since the problem is symmetric under the exchange $m \leftrightarrow n$, it is sufficient to consider lines containing $(2,0),(2,1)$ or $(3,0)$. This geometrical division into cases corresponds exactly with the classification of Arnold. Figure 1 shows the lines containing $(2,0)$ or $p^{2}$

$$
H(p, q)= \begin{cases}A p^{2}+C q^{N}, & N \geqq 3, \text { odd } \\ A p^{2}+B p q^{N / 2}+C q^{N}, & N \geqq 4, \text { even }\end{cases}
$$

Figure 2 pictures those containing $(2,1)$ or $p^{2} q$

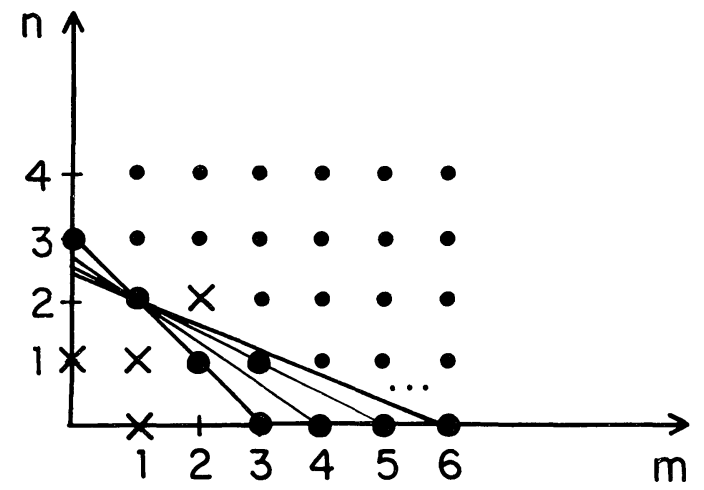

Fig. 2. Arnold's simple singularities $D_{N}$ 


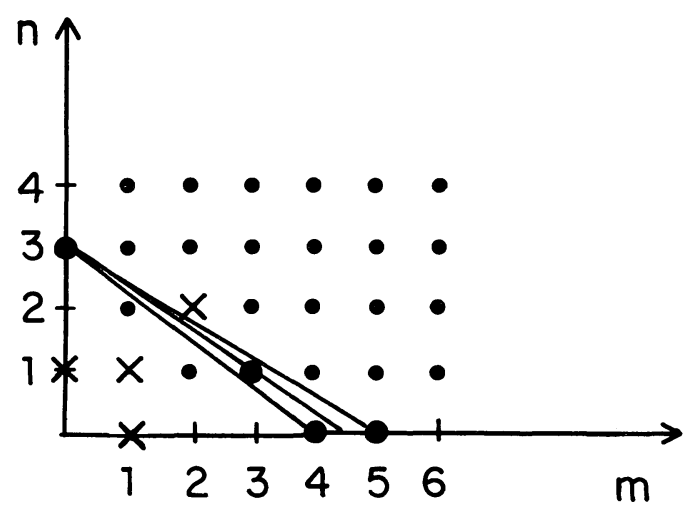

Fig. 3. Arnold's simple singularities $E_{N}$

$$
H(p, q)=\left\{\begin{array}{ll}
D p^{3}+A p^{2} q+B p q^{2}+C q^{3}, & \\
A p^{2} q+C q^{N}, & N \geqq 4, \text { even } \\
A p^{2} q+B p q^{N+1 / 2}+C q^{N}, & N \geqq 5, \text { odd }
\end{array} .\right.
$$

Figure 3 shows those containing $(3,0)$ or $p^{3}$

$$
H(p, q)=\left\{\begin{array}{l}
A p^{3}+C q^{4} \\
A p^{3}+C p q^{3} \\
A p^{3}+C q^{5}
\end{array}\right.
$$

The list may be simplified via canonical transformation. All terms with coefficients $B$ or $D$ may be eliminated. The absence of lines of fixed points ensures that the new values of $A$ and $C$ will not be zero. Another canonial transformation makes $A= \pm C$, so that all solutions are a scalar multiple of one of the basic solutions

$$
H(p, q)= \begin{cases}p^{2} \pm q^{N}, & \lambda=\frac{2 N}{(N-2)}, \quad N \geqq 3 \\ p^{2} q \pm q^{N}, & \lambda=\frac{2 N}{(N-1)}, \quad N \geqq 3 \\ p^{3}+q^{4}, & \lambda=\frac{12}{5} \\ p^{3}+p q^{3}, & \lambda=\frac{9}{4} \\ p^{3}+q^{5}, & \lambda=\frac{15}{7} .\end{cases}
$$

These are the simple singularities of Arnold, called $A_{N}, D_{N}$ and $E_{N}$ [3].

\section{Physical Consequences}

A scheme of classification is only valuable when it results in a significant limitation of possible behaviors. There are two criteria which determine which normal forms of Eq. (27) are actually relevant to the physical problem of intermittency. The first 
criterion is the existence of a perturbation which eliminates the fixed point. The condition that two eigenvalues be equal to one was necessary, but as will be shown, not sufficient to guarantee the existence of such a perturbation. The relevance of a solution may also be evaluated by counting the number of relevant perturbations to it. Each of these corresponds to a parameter which must be varied to achieve criticality. Experimental observation becomes difficult when there are very many of them. Once the set of relevant solutions is determined, it only remains to describe the behavior in each case. This is done by calculating the effects of relevant perturbations on the basic quantity $\tau$.

There is a simple relation between the winding number of a vector field and the existence of perturbations which eliminate its fixed points. The winding number of a vector field in some bounded region is $1 /(2 \pi)$ times the net change in the argument of the field along a path enclosing the zeroes of the field [22]. This is an important characterization because it is invariant under perturbation. For example, the winding number of a vector field with a single elliptic or hyperbolic fixed point is +1 or -1 , respectively. The winding number of a field in a region with no fixed points is equal to zero. Thus only those fields with winding number zero may lose all fixed points through perturbation.

The winding numbers of the flows defined by the Hamiltonians of Eq. (27) are easy to calculate. The answer turns out to depend only on the number of branches of the solution set of the equation $H(p, q)=0$. Each branch of the set $H(p, q)=0$ is a trajectory which approaches the fixed origin as $t \rightarrow+\infty$ or as $t \rightarrow-\infty$. Following a circular path around the fixed origin, the flow direction on each branch is opposite to that of the previous branch (because the flow is divergence-free). If there are $n$ branches, the argument changes by $(2 \pi-n \pi)$ during one complete revolution and the winding number is equal to $(1-n / 2)$. So there exist perturbations removing the fixed points only when $H(p, q)=0$ has two branches. In each of these cases it is easy to find such a perturbation explicitly. The following Hamiltonians generate flows with no fixed points (when $\varepsilon>0$ )

$$
H(p, q)= \begin{cases}p^{2}+q^{n}+\varepsilon q, & n \geqq 3, n \text { odd } \\ p^{2} q+q^{n}+\varepsilon q, & n \geqq 3, n \text { odd } \\ p^{3}+q^{4}+\varepsilon p, & \\ p^{3}+q^{5}+\varepsilon q . & \end{cases}
$$

Thus only these Hamiltonians (with $\varepsilon=0$ ) may be the leading behavior when a fixed point disappears.

The likelihood of observing a given scaling form depends on its codimension, defined as the number of independent relevant perturbations. There is an exact correspondence between the codimension defined in this way and that defined by Arnold in terms of "versal deformations" [3]. The method of calculating the codimension will be illustrated for the series $H(p, q)=p^{2} \pm q^{N}$. All relevant perturbations are included in the perturbed form

$$
H(p, q)=p^{2} \pm q^{N}+p f(q)+\sum_{k=0}^{N-1} a_{k} q^{k}
$$


where $f(q)$ is a polynomial of degree [N/2]. The canonical transformation $(p, q) \rightarrow(p-1 / 2 f(q), q)$ removes the term $p f(q)$ from the Hamiltonian while changing the coefficients $a_{k}$. Then the canonical transformation $(p, q) \rightarrow\left(p, q-(1 / N) a_{N-1}\right)$ removes the term $a_{N-1} q^{N-1}$. Any constant $a_{0}$ may be removed by the canonical transformation $H(p, q) \rightarrow H(p, q)-a_{0}$. This leaves the $(N-2)$ relevant perturbations $q, q^{2}, \ldots, q^{N-2}$. The independence of these perturbations may be verified by an analysis of the effects of infinitesimal canonical transformations. Thus the codimension is $(N-2)$. A similar analysis gives the $N$ independent relevant perturbations $p, q, q^{2}, \ldots, q^{N-1}$ to the Hamiltonian $H(p, q)=p^{2} q+q^{N}$, which therefore has codimension $N$. The Hamiltonians $H(p, q)=p^{3}+q^{4}, p^{3}+p q^{3}$ and $p^{3}+q^{5}$ have codimension 5, 6 and 7, respectively. If one assumes that experimenters have at most four independent knobs with which to control parameters, the list of possible leading behaviors at the intermittency threshold is quite short

$$
H(p, q)=\left\{\begin{array}{l}
p^{2}+q^{3} \\
p^{2}+q^{5} \\
p^{2} q+q^{3} .
\end{array}\right.
$$

These leading behaviors are of codimension 1,3 and 3 respectively. Being of codimension one, the form $p^{2}+q^{3}$ is "generic" and will arise naturally in cases with no additional symmetry.

The final task for theory is to calculate $\tau$ in the presence of perturbations. The behavior is dominated by the most relevant perturbation. Since $\Lambda=\lambda-\alpha n-\delta m$ and $\alpha \geqq \delta$, this perturbation is $\Delta H=q$ and has eigenvalue $\Lambda=\lambda-\delta=1+\alpha$. (Exception must be made for the case $H(p, q)=p^{3}+q^{4}+\varepsilon p$ where the perturbation $p$ has eigenvalue $\Lambda=1+\delta$.) As mentioned earlier, the effect of renormalization is to fix scaling Hamiltonians while slowing down orbits by a factor $e^{s}$. If $\varepsilon$ rescales as $e^{\Lambda s}$, then the perturbed Hamiltonian is also fixed by this renormalization. Since $\tau$ decreases as $e^{-s}$ while $\varepsilon$ increases as $e^{A s}$, it must be that

$$
\tau \propto \varepsilon^{-1 / \Lambda} .
$$

The most interesting potential application of this theory is to Hamiltonian systems with many degrees of freedom. When a stable periodic orbit disappears in such a system, the local behavior splits into two parts (in perturbation theory). One degree of freedom is marginal, in the sense of this paper, while the others are all elliptically stable. Thus one might expect the same behavior as in the one degree of freedom case (modulated by a small quasiperiodic function). Of course, due to Arnold diffusion, the perturbation theory breaks down on an exponentially long time scale. Nevertheless the phenomena described, which occur on a shorter powerlaw time scale, may be observable.

Note added in proof: $I$ wish to thank Prof. Michael V. Berry for bringing his interesting related work to my attention $[26,27]$. 


\section{References}

1. Birkhoff, G. D.: Dynamical Systems. Am. Math. Soc. Colloq. Pub., vol. 9 (1927)

2. Arnold, V. I.: Mathematical Methods of Classical Physics. Berlin, Heidelberg, New York: SpringerVerlag 1978

3. Arnold, V. I.: Funct. Anal. Appl. 6, 254 (1972)

4. Feigenbaum, M. J.: Quantitative universality for a class of nonlinear transformations, J. Stat. Phys. $19,25(1978)$

5. Feigenbaum, M. J.: The universal metric properties of nonlinear transformations, J. Stat. Phys. 21, 669 (1979)

6. Greene, J. M., MacKay, R. S., Vivaldi, F., Feigenbaum, M. J.: Universal behaviour in families of areapreserving maps, Physica 3D, 468 (1981)

7. Widom, M., Kadanoff, L. P.: Renormalization group analysis of bifurcations in area-preserving maps, Physica 5D, 287 (1982)

8. Kadanoff, L. P.: In: Melting, localization and chaos. eds. Kalia, R. K. Vashishta, P. (eds.), p. 209. New York: Elsevier Science Publishing Co., 1982

9. Zisook, A. B.: Intermittency in area-preserving mappings, Phys. Rev. A25, 2289 (1982)

10. Zisook, A. B., Shenker, S. J., Renormalization group for intermittency in area-preserving mappings, Phys. Rev. A25, 2824 (1982)

11. Hu, B., Rudnick, J.: Exact solutions to the renormalization-group fixed-point equations for intermittency in two-dimensional maps, Phys. Rev. A26, 3035 (1982)

12. Manneville, P., Pomeau, Y.: Intermittency and the Lorenz model, Phys. Lett. 75A, 1 (1979)

13. Manneville, P., Pomeau, Y.: Intermittent transition to turbulence in dissipative dynamical systems, Commun. Math. Phys. 74, 189 (1980)

14. Dubois, M., Rubio, M. A., Bergé, Experimental evidence of intermittencies associated with a subharmonic bifurcation P.: Phys. Rev. Lett. 51, 1446 (1983)

15. Gibson, G., Jeffries, C.: (submitted for publication)

16. Hirsch, J. E., Huberman, B. A., Scalapino, D. J.: Theory of intermittency, Phys. Rev. A25, 519 (1982)

17. Hirsch, J. E., Nauenberg, M., Scalapino, D. J.: Intermittency in the presence of noise: A renormalization group formulation, Phys. Lett. 87A, 391 (1982)

18. Hu, B., Rudnick, J.: Exact solutions to the Feigenbaum renormalization-group equations for intermittency, Phys. Rev. Lett. 48, 1645 (1982)

19. Arnold, V. I., Avez, A.: Ergodic problems of classical mechanics. New York, Amsterdam: Benjamin, 1968

20. Chirikov, B.: A universal instability of many-dimensional oscillator systems, Phys. Rep. 52, 265 (1979)

21. Nonlinear dynamics and the beam-beam interaction. Month M., Herrera, J. C. (eds.), Vol. 57. Am. Inst. Phys. Conf. Proc. (1979)

22. Guilleman, V., and Pollack, A.: Differential topology. New Jersey: Prentice Hall, 1974

23. Mackay, R. S.: A renormalization approach to invariant cycles in area-preserving maps, Physica 7D, $283(1983)$

24. Collet, P., Eckmann, J.-P., Koch, H.: On universality for area-preserving maps of the plane, Physica 3D, 457 (1981)

25. Eckmann, J.-P., Koch, H., Wittwer, P.: Existence of a fixed point of the doubling transformation for area-preserving maps of the plane, Phys. Rev. A26, 720 (1982)

26. Walker, J. G., Berry, M. V., Upstill, C.: Measurement of twinkling exponents of light focused by randomly rippling water, Optica Acta 30, 1001 (1983)

27. Berry, M. V.: Universal power-law tails for singularity-dominated strong fluctuations, J. Phys. A 15, 2735 (1982)

Communicated by O. E. Lanford

Received January 20, 1984; in revised form June 14, 1984 
